\title{
Cell Strain
}

National Cancer Institute

\section{Source}

National Cancer Institute. Cell Strain. NCI Thesaurus. Code C45382.

Cells adapted to culture, but with finite division potential. 